\title{
Neonatal platelets: mediators of primary hemostasis in the developing hemostatic system
}

\author{
Kristina M. Haley ${ }^{1}$, Michael Recht ${ }^{1}$ and Owen J.T. McCarty ${ }^{2}$
}

The human hemostatic system is developmentally regulated, resulting in qualitative and quantitative differences in the mediators of primary and secondary hemostasis as well as fibrinolysis in neonates and infants. Although gestational age-related differences in coagulation factor levels occur, the existence of a unique neonatal platelet phenotype remains controversial. Complicated by difficulties in obtaining adequate neonatal blood volumes with which to perform functional assays, ambiguity surrounds the characterization of neonatal platelets. Thus, much of the current knowledge of neonatal platelet function has been based on studies from cord blood samples. Studies suggest that cord blood-derived platelets, as a surrogate for neonatal platelets, are hypofunctional when compared with adult platelets. This relative platelet dysfunction, combined with a propensity toward thrombocytopenia in the neonatal intensive care unit population, creates a clinical conundrum regarding the appropriate administration of platelet transfusions. This review provides an appraisal of the distinct functional phenotype of neonatal platelets. Neonatal platelet transfusion practices and the impact of the relatively hypofunctional neonatal platelet on those practices will be considered.

$\mathbf{T}$ he hemostatic system is maintained by a delicate balance of pro- and anticoagulant factors, allowing for physiologic response to hemorrhage while simultaneously avoiding development of pathologic thrombi. This balance of opposing forces can be further broken down into primary hemostasis, secondary hemostasis, and fibrinolysis. Primary hemostasis, the formation of a platelet plug, is mediated by the interaction of platelets and disrupted or damaged endothelium lining blood vessels. Secondary hemostasis, the generation of thrombin resulting in the cleavage of fibrinogen to fibrin, is an orchestrated event of sequential amplification of enzymatic activation. Cessation of bleeding occurs through the coordinated efforts of primary and secondary hemostasis. To prevent the formation of pathologic thrombi and restrict clot formation to the area of injury, the anticoagulant system is nearly simultaneously activated in order to limit clot extension and maintain its boundaries to the damaged area until the damage can be repaired.

The neonatal hemostatic system is both quantitatively and qualitatively distinct from that of an adult. The term "developmental hemostasis" has been applied to the period of time when the neonatal hemostatic system exists in an evolving balance of pro- and anticoagulant factors $(1,2)$. The components of the neonatal secondary hemostatic system mature in a predictable way. Reference ranges have been determined for both pro- and anticoagulant proteins for neonates at different gestational ages as well as infants. Furthermore, normal ranges for global assessments of the secondary hemostatic system such as the activated partial thromboplastin time or the prothrombin time have also been determined for neonates and infants. After 6 mo of age, adult reference ranges can be used to determine if a particular value is normal (2).

The task of defining age-dependent normal values of neonatal platelet function has been fraught with challenges (1). Reference ranges for platelet counts in neonates and adults are similar $(3,4)$. However, developmental changes and the impact of those changes on the functional aspects of primary hemostasis are less clear than that in the secondary hemostatic system. Assessment of platelet function, regardless of age, is challenging as platelet function tests often require large volumes of blood, demand specialized laboratories and expertise, and poorly simulate in vivo primary hemostasis. Currently, the neonatal platelet is characterized as hypofunctional as compared with the adult platelet despite evidence of more efficient global primary hemostasis in neonates (5). In this review, the literature regarding neonatal platelet function will be reviewed and synthesized to more clearly define the neonatal platelet functional phenotype and its contribution to the newborn's hemostatic system (Table 1; refs. (6-18)).

\section{NORMAL PLATELET FUNCTION \\ Platelet Adhesion}

Following vascular injury and the attendant endothelial damage, platelet adhesion, initiating the process of primary hemostasis, is mediated through receptor/ligand interactions in a stepwise manner $(19,20)$ : 


\section{Neonatal platelet function $\quad$ Review}

Table 1. Summary of selected neonatal platelet function literature

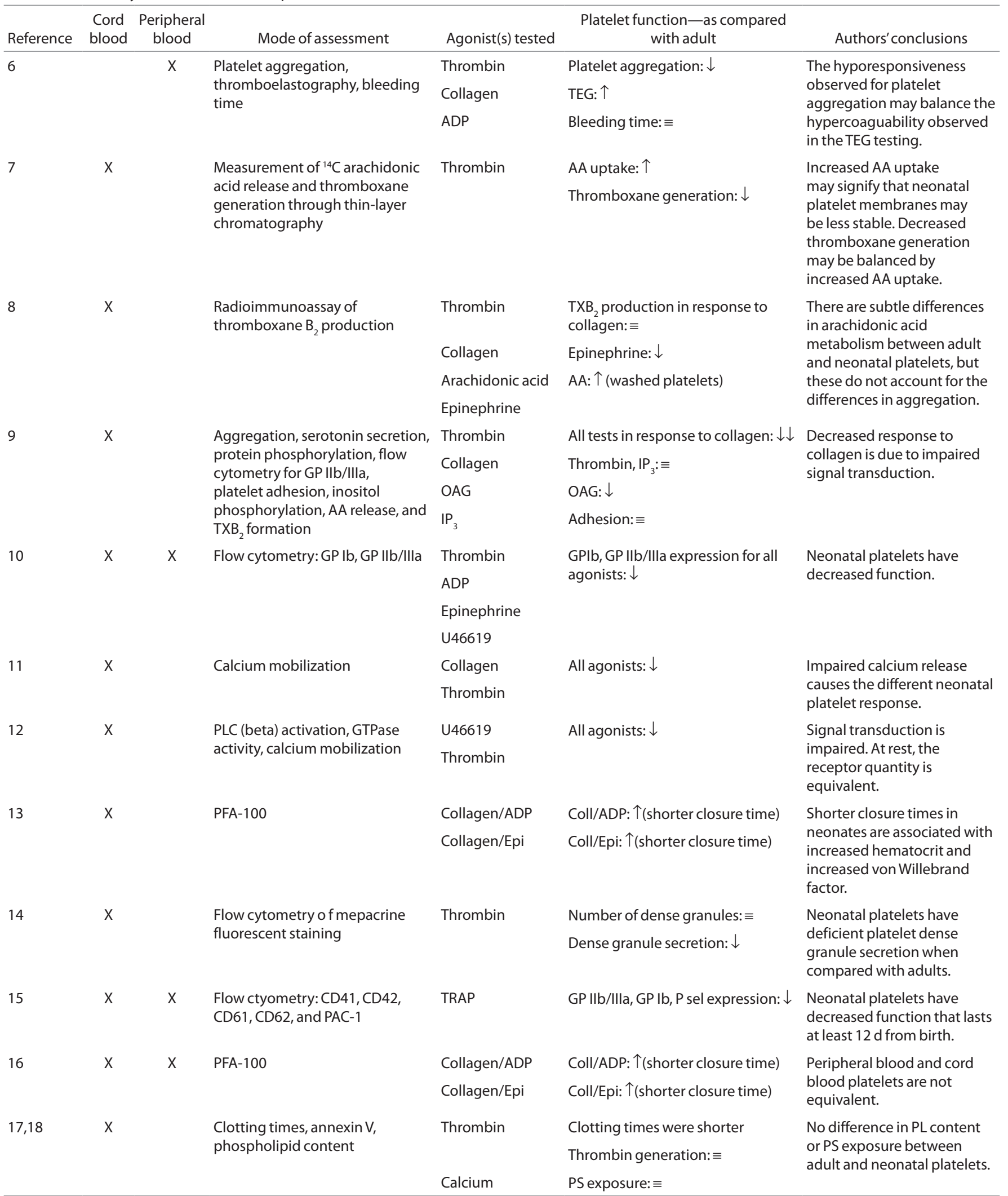

Publications are listed by first author and year of publication. Type of blood used for neonatal samples is listed as either cord blood or peripheral blood. The results of the platelet function testing with regard to neonatal vs. adult platelet function is noted: $\uparrow$ denotes that neonatal platelet responsiveness exceeds adult platelet responsiveness, $\downarrow$ denotes that neonatal platelet responsiveness is less than adult platelet responsiveness, and $\equiv$ denotes that neonatal and adult platelet responsiveness are equivalent.

ADP, adenosine diphosphate; PL, phospholipid; PLC, phospholipase C; PS, phosphatidylserine; TEG, thromboelastography; TRAP, thrombin receptor agonist peptide . 
- Extracellular von Willebrand factor (VWF)-platelet glycoprotein (GP) Ib binding mediates initial platelet recruitment to the injured area.

- Platelet GPVI interacts with fibrillar collagen and platelet $\beta_{1}$ integrin interacts with laminin, collagen, and fibronectin allowing for firm adhesion of platelets to the exposed extracellular matrix.

\section{Platelet Activation}

Following platelet adhesion, a series of downstream signaling events results in an increase in intracellular calcium and subsequent platelet activation, marked by (Figure 1):

1. Exposure of negatively charged phosphatidylserine (PS) on the platelet membrane surface, allowing for the assembly of coagulation factors;

2. Platelet alpha and delta granule secretion resulting in the release of adenosine diphosphate (ADP), calcium, serotonin, VWF, coagulation factors V and VIII, and fibrinogen;

3. Platelet membrane GPIIb/IIIa integrin conversion to a high-affinity state for VWF and fibrinogen binding;

4. Thromboxane $\mathrm{A}_{2}$ generation through arachidonic acid metabolism;

5. Cytoskeletal reorganization to increase the surface area of spread platelets.

\section{Platelet Aggregation}

A key step for the development of a stable platelet aggregate is the conversion of the GPIIb/IIIa receptor into its high-affinity conformation. This allows for stable interactions between the receptor and fibrinogen, VWF, and fibronectin. Platelets aggregate together, forming a platelet plug-the end product of primary hemostasis (19).

In summary, primary hemostasis is achieved through a synergistic network of receptor/ligand interactions that result in platelet adhesion and simultaneous platelet activation, platelet secretion to activate nearby platelets, platelet aggregation, and ultimately formation of a platelet plug and generation of a surface amenable to assembly of coagulation factor complexes.

\section{PLATELET FUNCTION TESTING}

Platelet function testing is notoriously challenging, owing to difficulties in replicating the physiologic circumstances under which primary hemostasis occurs (Table 2) (21). Typically, clinical evaluation of platelet function begins with a complete blood count and review of the peripheral smear. This assesses for adequate platelet number as well as platelet size and alpha granule content. Platelet aggregation is most commonly assessed via aggregometry, in which light transmission through a platelet suspension is measured. An initial increase in optical density correlates with platelet shape change and sphericity, and the decrease in optical density indicates platelet aggregation in response to known platelet agonists. The primary limitation with platelet aggregometry is the volume of blood needed for the test, which is typically $10-15 \mathrm{ml}$ to test an adequate panel of agonists. The development of whole-blood aggregometers that employ small volumes of blood addresses this challenge. Clinical application is limited due to lack of neonatal reference ranges and limited availability of the technology (22). The platelet function analyzer, PFA-100, simulates primary hemostasis through the aspiration of anticoagulated whole blood through a narrow aperture in a membrane coated with collagen and ADP or collagen and epinephrine. Time to occlusion of the membrane, or closure time, is the measurement output. The advantage of the PFA-100 is the small amount of blood needed to run the test ( $800 \mu \mathrm{l}$ per cartridge). The limited specificity and sensitivity of the PFA-100 for detecting qualitative platelet defects limits the utility of this technique for neonatal platelet studies. Flow cytometry makes use of fluorophore-conjugated antibodies to platelet receptors such as GPIb-IX-V (low or absent in Bernard-Soulier syndrome) or GPIIb/IIIa (low or absent in Glanzmann's thrombasthenia) in order to quantify the extent of surface membrane protein expression. Flow cytometry can be employed to assess platelet function through detection of P-selectin expression or activated GPIIb/IIIa; however, the use of flow cytometry in this functional way has not been fully explored in clinical use. Flow cytometry holds significant promise for neonatal platelet function testing, as minimal blood is required for this testing. Electron microscopy allows for examination of platelet cytoskeletal structure and quantification of platelet dense granules at a single platelet level $(21,23)$.

\section{NEONATAL PLATELET FUNCTION}

The challenges associated with defining neonatal platelet function exist on several levels (21). First, obtaining adequate blood volume to fully evaluate neonatal platelet function is challenging, especially at early gestational ages. Second, it is unclear how other hemostatic variables influence platelet function. More specifically, it has not been well defined how altered levels of pro- and anticoagulant proteins influence platelet function. Neonates frequently have a higher hematocrit as well as higher mean corpuscular volumes than older children and adults (3), which likely enhance primary hemostasis and may offset the altered function of the neonatal platelet. Current available testing methods are less than ideal and typically not feasible in the neonatal population $(1,24)$.

Neonatal platelet function has been assessed at each step in primary hemostasis. The majority of the studies have used platelets derived from cord blood rather than from peripheral blood samples for functional analysis. The summation of this data has led to the designation of neonatal platelets as hyporeactive $(1,3,24)$. The availability and accessibility of cord blood makes it an often-employed substitute for peripherally obtained blood for the majority of the studies aimed at elucidating the neonatal platelet phenotype. However, it is unclear if the function of cord blood-derived platelets accurately represents neonatal platelet function (16).

As described above, a platelet plug is formed in response to vascular injury following platelet adhesion, activation, 


\section{Neonatal platelet function $\quad$ Review}

and aggregation. Neonatal whole blood, subjected to shear, has been shown to produce a significantly higher deposition of platelets on extracellular matrix proteins and VWF when compared with adult samples. This finding was true for both cord blood- and peripheral blood-derived platelets (25). Thus, whether the hyporeactivity of neonatal platelets translates to a diminished hemostatic response is unclear.

Platelet activation, at each of the steps described in Figure 1, has been evaluated and led to the designation of the neonatal

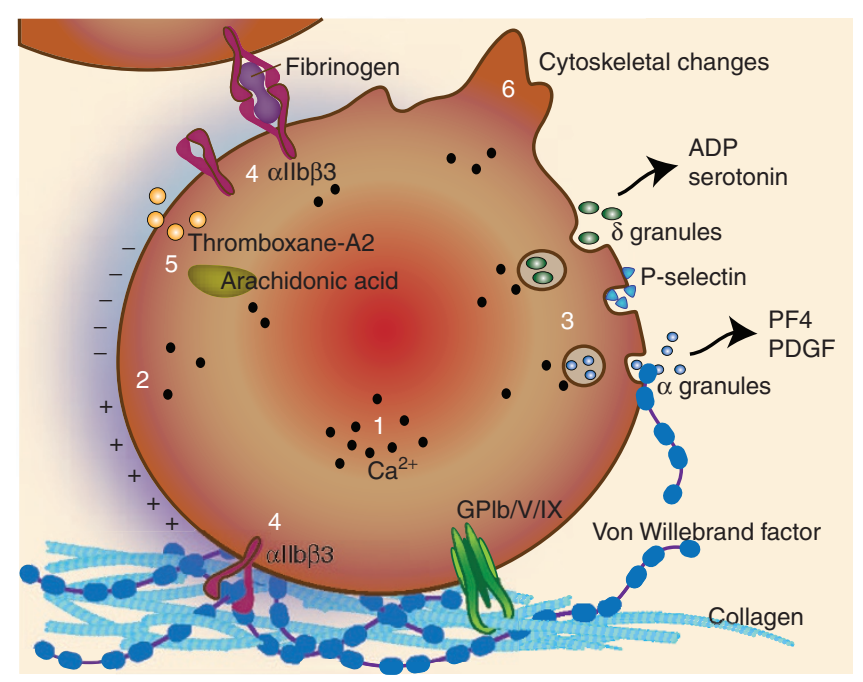

Figure 1. The mechanisms of platelet adhesion, activation, and secretion. The platelet receptor complex GPIb/V/IX mediates platelet recruitment to von Willebrand factor bound to exposed extracellular matrix proteins, such as collagen. Subsequent platelet activation results in an increase in intracellular calcium (1), increased phosphatidylserine exposure to create a negatively charged surface (2), alpha and dense granule release (3), conversion of the GPIIb/IIla receptor to a high-affinity state (4), thromboxane A2 generation (5), and cytoskeletal rearrangement (6). ADP, adenosine diphosphate. platelet as hypofunctional. Evaluating each aspect of platelet activation allows for critical appraisal of this designation.

1. PS exposure: Annexin V, which binds PS with high affinity, can be used to characterize the procoagulant phenotype of activated platelets. Analysis of PS exposure through measurement of Annexin V binding via flow cytometry has shown that the procoagulant phenotype is similar for both cord blood-derived platelets and adult platelets following stimulation with thrombin $(17,18)$. Along these lines, phospholipid content of adult and cord blood platelets has also been reported to be similar $(17,18)$. The procoagulant phenotype of peripheral blood-derived neonatal platelets has yet to be defined.

2. Granule content and release: Platelet activation is further marked by alpha and delta (dense) granule release. Release of granular contents further promotes autocrine and paracrine stimulation, strengthening platelet adhesion and activating nearby platelets. P-selectin expression is greatly enhanced on the platelet surface following activation and alpha granule secretion; therefore, antibodies directed at P-selectin (anti-CD62P) are used as markers of alpha granule release, and, hence, platelet activation. P-selectin expression following thrombin stimulation on platelets derived from neonatal peripheral blood and cord blood has been compared with adult platelet P-selectin expression. Following stimulation, P-selectin expression was lower in neonatal platelets obtained from both cord blood and peripheral blood as compared with adult platelets $(10,15)$. This difference remained statistically significant up to day 12 of life, and this difference was greatest between neonates less than $30 \mathrm{wk}$ of gestation and adults (15). No difference

Table 2. Clinically used tests of platelet function and the potential clinical implications of abnormal results

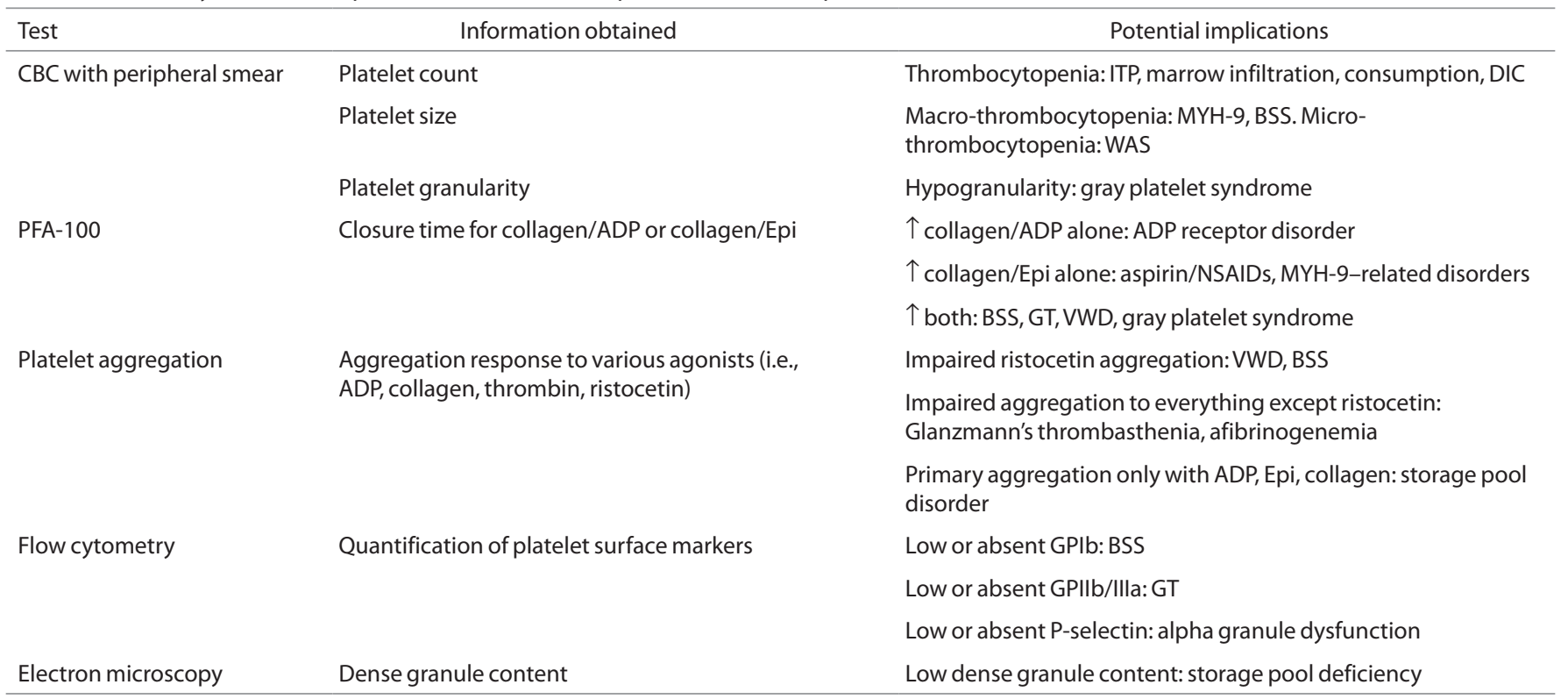

ADP, adenosine diphosphate; BSS, Bernard-Soulier syndrome; CBC, complete blood count; DIC, disseminated intravascular coagulation; GT, Glanzmann's thrombasthenia; ITP, immune thrombocytopenic purpura; MYH, myosin heavy chain; NSAIDs, nonsteroidal anti-inflammatory drugs; VWD, von Willebrand disease; WAS, Wiskott-Aldrich syndrome. 
was reported between neonatal platelet (derived from either cord or peripheral blood) and adult platelet delta granule release, as assessed by measurement of serotonin secretion, in response to stimulation with thrombin or the platelet agonists 1-oleolyl-2-acetyl-glycerol and inositol triphosphate $\left(\mathrm{IP}_{3}\right)$. However, serotonin secretion was markedly decreased in cord blood-derived neonatal platelets in response to collagen as compared with adult platelets (9). Furthermore, as measured by mepacrine uptake, neonatal platelets (from cord blood) contained the same number of dense granules as adult platelets, but these same platelets had defective release of dense granules following thrombin stimulation (14). Taken together, these studies suggest that granule secretion may be functionally different between neonatal and adult platelets.

3. Glycoprotein IIb/IIIa conversion to a high-affinity state: The conversion of the platelet surface receptor GPIIb/IIIa to its high-affinity state is a critical step in the development of a platelet aggregate and allows for the formation of fibrinogen bridges between adjacent platelets. The active form of GPIIb/IIIa can be assessed by flow cytometry through assessment of binding of PAC-1, an antibody that only recognizes the active form of GPIIb/ IIIa. GPIIb/IIIa baseline expression has been reported to be equivalent for neonatal (cord and peripheral) and adult platelets (15). However, the percent of PAC-1-positive platelets following stimulation with thrombin was lower in neonatal samples (cord and peripheral) as compared with adult samples (15). These studies suggest that the signaling pathways that regulate GPIIb/IIIa activation may be regulated differently in neonatal vs. adult platelets.

4. Thromboxane generation: Generation and release of thromboxane $\mathrm{A}_{2}\left(\mathrm{TxA}_{2}\right)$ via arachidonic acid metabolism allows for stimulation of nearby platelets, enhancing platelet adhesion, activation, and ultimately aggregation. Thromboxane $\mathrm{B}_{2}\left(\mathrm{TxB}_{2}\right)$ is a metabolite of $\mathrm{TxA}_{2}$ and frequently used as a marker of $\mathrm{TxA}_{2}$ generation. Early studies demonstrated that cord blood-derived neonatal platelets release more arachidonic acid in response to thrombin when compared with adult platelets; however, the cord blood platelets synthesized less $\mathrm{TxB}_{2}$ (7). Subsequent studies have failed to find a difference in cord blood-derived neonatal platelet $\mathrm{TxB}_{2}$ generation in response to thrombin and collagen when compared with adult platelet $\mathrm{TxB}_{2}$ generation (8). In platelet-rich plasma, cord blood-derived neonatal platelets produced a greater amount of $\mathrm{TxB}_{2}$ as compared with adult platelets in response to arachidonic acid (8). This difference reversed after platelet washing, suggesting that factors present in the plasma may account for this difference (8). Lastly, cord blood-derived neonatal platelets produced less $\mathrm{TxB}_{2}$ in response to epinephrine as compared with adult platelets (8). Taken together, these studies suggest that thromboxane production is differently regulated in neonatal platelets as compared with adult platelets.
5. Platelet cytoskeletal reorganization: Upon platelet activation, significant morphologic rearrangement occurs resulting in the platelet taking on a "fried egg" appearance, thus increasing its exposed surface area. The ultrastructure of unstimulated cord blood-derived neonatal platelets is similar to that of adult platelets. Differences in pseudopod number, microtubular structures, and granular content have been demonstrated, but there is concern that the differences were secondary to sampling technique $(26,27)$. Morphologic changes following activation have not been investigated in the neonatal platelet.

The differences described above have been attributed to alterations in calcium mobilization and intracellular signaling within neonatal platelets as compared with adult platelets. Cord blood-derived neonatal and adult platelets had similar levels of baseline calcium (11). However, intracellular calcium release in neonatal platelets was reduced when compared with adult platelets in response to either collagen or thrombin. These differences were hypothesized to be secondary to a deficiency in $\mathrm{IP}_{3}$ production and interaction of $\mathrm{IP}_{3}$ with its receptor on the dense tubular system that ultimately is required for calcium release (11). Mobilization of calcium was found to be impaired in neonatal platelets in response to a thromboxane analogue. Furthermore, $G$ protein signaling was found to be decreased in neonatal platelets as compared with adult platelets, which may provide a mechanism explaining the reduced levels of activation observed for neonatal platelets (12).

\section{CLINICALLY BASED ASSAYS OF NEONATAL PLATELET FUNCTION}

In a 1970 pioneering study, neonatal platelets from cord and peripheral blood were found to have defective aggregation in response to $\mathrm{ADP}$, collagen, or thrombin. Using thrombelastography, neonatal blood has demonstrated contrasting hypercoagulability with shortened $\mathrm{r}+\mathrm{k}$ times $(r=$ reaction time to initial fibrin formation, $k=$ time to cross link fibrin) and increased maximal amplitude, a reflection of clot strength and the combination of fibrin formation, fibrin cross-linking, and platelet aggregation. The platelet "transient hyporesponsiveness" was hypothesized to be an important defense mechanism against pathologic thrombus formation during a time when an infant may be hypercoagulable (6). Thirty years later, whole cord blood was shown to have significantly shorter PFA-100 closure times as compared with whole blood samples from adults $(13,16,28)$. Interestingly, closure times were significantly longer in whole peripheral blood obtained from healthy neonates in the first week of life than in cord blood samples, suggesting increased primary hemostatic activity in the cord blood samples (16). The shorter closure times in neonatal samples in addition to findings of shorter or similar bleeding times in neonates as compared with adults (29) suggests enhanced primary hemostasis. Recently, PFA closure times have been evaluated in preterm neonates with thrombocytopenia. No association with platelet count and epinephrine closure times was found, but the ADP closure time was frequently 


\section{Neonatal platelet function}

\section{Review}

more prolonged in thrombocytopenic infants (30). This study emphasized differences between premature and full-term neonatal platelet function and also highlighted the challenge of lack of neonatal normal values and the influence of concomitant thrombocytopenia on functional testing.

Bleeding times have largely fallen out of favor due to interoperator variability and inconsistent reference ranges. However, a large study evaluating a modified bleeding time demonstrated that the bleeding time shortens between day of life 1 and 10 (31). Additional studies comparing bleeding times have demonstrated shorter times in neonates than adults (29). Enhanced primary hemostasis is thought to be secondary to increased presence of higher molecular weight VWF, higher concentrations of VWF, higher hematocrit, and larger red blood cell mean corpuscular volume in cord and peripheral neonatal blood samples $(13,16)$ that collectively potentially mitigate the relative hypofunctional nature of the neonatal platelet.

\section{DEVELOPMENT OF A NEW FUNCTIONAL ASSAY}

As assessment of neonatal platelet function is fraught with challenges related to blood volume, we are developing a smallvolume assay for characterization of neonatal platelet function in whole-blood samples. The hAPI (the Assessment of Platelet Interactions) assay is designed to require less than $1 \mathrm{ml}$ of whole blood in total to simultaneously assess platelet adhesion, activation, and aggregation. Anticoagulated whole blood obtained via heel stick is placed onto glass slides coated with either collagen or fibrinogen. A representative image of neonatal platelets (Figure 2) indicates that the neonatal platelet is capable of adhering to and spreading on surfaces of fibrinogen or collagen, resulting in secretion of alpha granules (as evidenced by P-selectin exposure). Future work will evaluate differences in platelet function at varying gestational ages and known platelet defects.

\section{PLATELET TRANSFUSIONS IN THE NEONATE}

The unique hypofunctional phenotype attributed to cord blood neonatal platelets plays an unclear role in disruptions in neonatal hemostatic balance. The neonatal intensive care unit population is at substantial risk for both hemorrhagic and thrombotic complications. Neonates have the highest risk of intracranial hemorrhage of any age group: $25-31 \%$ of preterm infants born with a birth weight less than $1,500 \mathrm{~g}$ experience some degree of intraventricular hemorrhage (IVH) (3). The role of neonatal platelet hyporeactivity in the development of IVH is unclear. IVH pathogenesis is thought to be multifactorial, attributed to fragility of the germinal matrix vessels, disturbances in cerebral blood flow, and platelet/coagulation disorder (32).

Confounding the potential for clinical complications resulting from differences in hemostasis is a high prevalence of thrombocytopenia in the neonatal population, particularly in the premature population. Thrombocytopenia (platelet counts less than $150 \times 10^{9} / 1$ ) affects up to $30 \%$ of all neonatal intensive care unit patients and $70 \%$ of those patients with a birth weight of less than $1,000 \mathrm{~g}(3,33)$. In response to thrombocytopenia

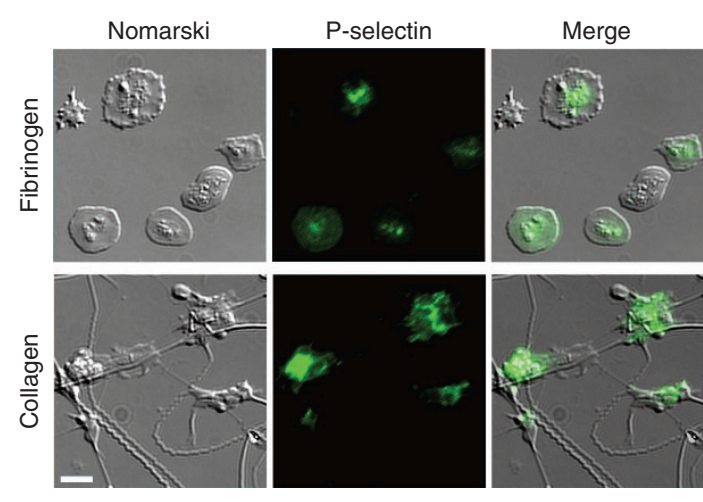

Figure 2. The Assessment of Platelet Interactions (hAPI) assay is being developed to characterize the functional phenotype of neonatal platelets obtained directly from the neonate. A representative neonatal sample is depicted here. Anticoagulated whole blood obtained from a newborn via heel stick was layered over glass slides coated with collagen or fibrinogen. The degree of platelet adhesion and spreading was monitored by differential interference contrast microscopy (Nomarski microscopy). P-selectin expression was determined through the binding of a fluorescein isothiocyanate-conjugated anti-P-selectin antibody (P-selectin), allowing for the assessment of P-selectin-positive platelets (merge). Images are at original magnification: $\times 63$. Bar $=10 \mu \mathrm{m}$.

and the high risk of IVH in this population, transfusion of platelets is a common intervention despite a clear lack of evidence for this practice (3). Transfusion practices vary widely internationally, with US neonatologists utilizing more liberal transfusion parameters than neonatologists outside the United States (34). Approximately 5-9\% of neonatal intensive care unit patients will receive at least one platelet transfusion during their stay $(35,36)$, and $50 \%$ of those patients receive two or more platelet transfusions $(34,37)$. The majority of transfusions, up to $95 \%$, are given prophylactically to neonates who are not bleeding with platelet counts ranging from $50-150 \times 10^{9} / 1$ (38), a much higher value than what is tolerated for older children and adults. Prophylactic platelet transfusions are often employed due to the concern about the development of IVH in this predisposed population, however; a causal relationship between mild-to-moderate thrombocytopenia and IVH in preterm infants has not been established, and prophylactic transfusions do not necessarily influence the extent or severity of IVH (39).

As in other populations, the transfusion of platelets to neonates comes with the potential for significant deleterious effects. Recent evidence has suggested an increased mortality rate in those neonates receiving platelet transfusions $(40,41)$. While a portion of this correlation can be attributed to comorbidities within the neonatal population, there is growing concern that platelet transfusions themselves have a negative effect on outcome $(38,41)$. Furthermore, platelet transfusions can be complicated by bacterial contamination, transfusion reactions, alloimmunization, allergic reactions, and transfusion-related lung injury. The lack of evidence guiding platelet transfusion practices in neonates has resulted in routine prophylactic transfusions to mitigate the theoretical contribution of presumed platelet hyporeactivity combined with thrombocytopenia in the development of intraventricular hemorrhage. The 
designation of cord blood neonatal platelets as hypofunctional by aggregation and some flow cytometry tests contradicts the evidence for effective primary hemostasis provided by the PFA-100 as well as clinical evidence that healthy newborns do not have bleeding complications. Furthermore, bleeding complications in premature newborns are not clearly linked to a hemostatic defect. Thus, the question remains: what is the role of the presumed neonatal platelet hyporeactivity to hemorrhagic complications in the neonatal period in premature or ill neonates where other hemostatic factors may be disrupted? Platelet transfusions are employed to counteract the perceived hypofunctionality and aim to protect against negative outcomes associated with hemorrhage, but the platelet transfusions themselves may be contributing to negative outcomes.

In studies designed to examine the effects of platelet transfusions on neonatal hemostasis, cord blood neonatal or adult platelets were mixed ex vivo with thrombocytopenic cord blood or adult blood, then evaluated with PFA-100 and thrombelastography. Closure times were significantly shorter in neonatal thrombocytopenic blood mixed with adult platelets compared with neonatal thrombocytopenic blood mixed with cord blood platelets (42). This study highlights the potential deleterious effect of transfusing adult platelets prophylactically into neonates with mild-to-moderate thrombocytopenia and the potential for potentiating a hypercoagulable, prothrombotic state.

\section{CONCLUSIONS}

In summary, the majority of neonatal platelet function studies have been conducted with cord blood rather than blood obtained directly from the neonate. It is not entirely clear that cord blood-derived platelets are an appropriate substitute for peripheral blood-derived platelets given the significant physiologic changes that occur following delivery. Furthermore, the available data regarding neonatal platelet function paints a picture of neonatal platelets that are hypofunctional in some regards but hyperfunctional in others. In isolation, cord bloodderived neonatal platelets have been found to have impaired calcium mobilization, GPIIb/IIIa activation, dense granule secretion, and alpha granule release and aggregation to a number of agonists as compared with adult platelets. However, bleeding times, PFA-100 closure times, and thromboelastography have all demonstrated a superior functionality of neonatal platelets as compared with adult platelets. Combined, these studies suggest that the neonatal platelet functional responses are different from adult platelets-much like other systems within the developing neonate-yet they are effective at its principal role of primary hemostasis. We propose the term "neonatelet" to describe this functionally distinct neonatal platelet. The duration that the neonatelet phenotype persists is unclear and how the maturation to the adult platelet occurs has yet to be determined. Further work is needed to more clearly define the neonatelet phenotype in healthy newborns as well as sick or premature newborns, as the characterization of the neonatelet may have important clinical implications in preventing and treating hemorrhage in the neonate as well as contributing to the development of platelet transfusion guidelines.

\section{ACKNOWLEDGMENTS}

The authors would like to thank Joe Aslan for his technical assistance.

\section{STATEMENT OF FINANCIAL SUPPORT}

This work was supported by the National Institutes of Health grant R01HL101972 (USA) (O.J.T.M.) and a Medical Research Foundation Early Clinical Investigator Award (Oregon, USA) (K.M.H.). O.J.T.M. is an American Heart Association Established Investigator (13EIA12630000) (USA).

Disclosure: The authors have no relevant conflict of interests to disclose.

\section{REFERENCES}

1. Strauss T, Sidlik-Muskatel R, Kenet G. Developmental hemostasis: primary hemostasis and evaluation of platelet function in neonates. Semin Fetal Neonatal Med 2011;16:301-4.

2. Kuhle S, Male C, Mitchell L. Developmental hemostasis: pro- and anticoagulant systems during childhood. Semin Thromb Hemost 2003;29:32938.

3. Sola-Visner M. Platelets in the neonatal period: developmental differences in platelet production, function, and hemostasis and the potential impact of therapies. Hematology Am Soc Hematol Educ Program 2012;2012:50611.

4. Kühne T, Imbach P. Neonatal platelet physiology and pathophysiology. Eur J Pediatr 1998;157:87-94.

5. Israels SJ, Rand ML, Michelson AD. Neonatal platelet function. Semin Thromb Hemost 2003;29:363-72.

6. Mull MM, Hathaway WE. Altered platelet function in newborns. Pediatr Res 1970;4:229-37.

7. Stuart MJ, Allen JB. Arachidonic acid metabolism in the neonatal platelet. Pediatrics 1982;69:714-8.

8. Stuart MJ, Dusse J, Clark DA, Walenga RW. Differences in thromboxane production between neonatal and adult platelets in response to arachidonic acid and epinephrine. Pediatr Res 1984;18:823-6.

9. Israels SJ, Daniels M, McMillan EM. Deficient collagen-induced activation in the newborn platelet. Pediatr Res 1990;27(4 Pt 1):337-43.

10. Rajasekhar D, Kestin AS, Bednarek FJ, Ellis PA, Barnard MR, Michelson $\mathrm{AD}$. Neonatal platelets are less reactive than adult platelets to physiological agonists in whole blood. Thromb Haemost 1994;72:957-63.

11. Gelman B, Setty BN, Chen D, Amin-Hanjani S, Stuart MJ. Impaired mobilization of intracellular calcium in neonatal platelets. Pediatr Res 1996;39(4 Pt 1):692-6.

12. Israels SJ, Cheang T, Roberston C, McMillan-Ward EM, McNicol A. Impaired signal transduction in neonatal platelets. Pediatr Res 1999;45(5 Pt 1):687-91.

13. Israels SJ, Cheang T, McMillan-Ward EM, Cheang M. Evaluation of primary hemostasis in neonates with a new in vitro platelet function analyzer. J Pediatr 2001;138:116-9.

14. Mankin P, Maragos J, Akhand M, Saving KL. Imparied platelet-dense granule release in neonates. J Pediatr Hematol Oncol 2000;22:143-7.

15. Sitaru AG, Holzhauer S, Speer CP, et al. Neonatal platelets from cord blood and peripheral blood. Platelets 2005;16:203-10.

16. Saxonhouse MA, Garner R, Mammel L, et al. Closure times measured by the platelet function analyzer PFA-100 are longer in neonatal blood compared to cord blood samples. Neonatology 2010;97:242-9.

17. Bernhard H, Rosenkranz A, Novak M, et al. No differences in support of thrombin generation by neonatal or adult platelets. Hamostaseologie 2009;29:Suppl 1:S94-7.

18. Bernhard H, Rosenkranz A, Petritsch M, et al. Phospholipid content, expression and support of thrombin generation of neonatal platelets. Acta Paediatr 2009;98:251-5.

19. Wei AH, Schoenwaelder SM, Andrews RK, Jackson SP. New insights into the haemostatic function of platelets. Br J Haematol 2009;147:415-30.

20. Aslan JE, McCarty OJ. Rho GTPases in platelet function. J Thromb Haemost 2013;11:35-46. 
21. Israels SJ, Kahr WH, Blanchette VS, Luban NL, Rivard GE, Rand ML. Platelet disorders in children: a diagnostic approach. Pediatr Blood Cancer 2011;56:975-83.

22. Halimeh S, Angelis Gd, Sander A, et al. Multiplate whole blood impedance point of care aggregometry: preliminary reference values in healthy infants, children and adolescents. Klin Padiatr 2010;222:158-63.

23. Cattaneo M. Inherited platelet-based bleeding disorders. J Thromb Haemost 2003;1:1628-36.

24. Israels SJ. Diagnostic evaluation of platelet function disorders in neonates and children: an update. Semin Thromb Hemost 2009;35:181-8.

25. Shenkman B, Linder N, Savion N, et al. Increased neonatal platelet deposition on subendothelium under flow conditions: the role of plasma von Willebrand factor. Pediatr Res 1999;45:270-5.

26. Saving KL, Jennings DE, Aldag JC, Caughey RC. Platelet ultrastructure of high-risk premature infants. Thromb Res 1994;73:371-84.

27. Saving K, Aldag J, Jennings D, Caughey B, Regan M, Powers W. Electron microscopic characterization of neonatal platelet ultrastructure: effects of sampling techniques. Thromb Res 1991;61:65-80.

28. Boudewijns M, Raes M, Peeters V, et al. Evaluation of platelet function on cord blood in 80 healthy term neonates using the Platelet Function Analyser (PFA-100); shorter in vitro bleeding times in neonates than adults. Eur J Pediatr 2003;162:212-3.

29. Andrew M, Paes B, Bowker J, Vegh P. Evaluation of an automated bleeding time device in the newborn. Am J Hematol 1990;35:275-7.

30. Deschmann E, Sola-Visner M, Saxonhouse MA. Primary hemostasis in neonates with thrombocytopenia. J Pediatr 2014;164:167-72.

31. Del Vecchio A, Latini G, Henry E, Christensen RD. Template bleeding times of 240 neonates born at 24 to 41 weeks gestation. J Perinatol 2008;28:427-31.

32. Ballabh P. Intraventricular hemorrhage in premature infants: mechanism of disease. Pediatr Res 2010;67:1-8.
33. Del Vecchio A, Motta M, Radicioni M, Christensen RD. A consistent approach to platelet transfusion in the NICU. J Matern Fetal Neonatal Med 2012;25:Suppl 5:93-6.

34. Cremer M, Sola-Visner M, Roll S, et al. Platelet transfusions in neonates: practices in the United States vary significantly from those in Austria, Germany, and Switzerland. Transfusion 2011;51: 2634-41.

35. Del Vecchio A, Sola MC, Theriaque DW, et al. Platelet transfusions in the neonatal intensive care unit:factors predicting which patients will require multiple transfusions. Transfusion 2001;41:803-8.

36. Christensen RD, Henry E, Wiedmeier SE, et al. Thrombocytopenia among extremely low birth weight neonates: data from a multihospital healthcare system. J Perinatol 2006;26:348-53.

37. Dohner ML, Wiedmeier SE, Stoddard RA, et al. Very high users of platelet transfusions in the neonatal intensive care unit. Transfusion 2009;49: 869-72.

38. Christensen RD. Advances and controversies in neonatal ICU platelet transfusion practice. Adv Pediatr 2008;55:255-69.

39. Andrew M, Vegh P, Caco C, et al. A randomized, controlled trial of platelet transfusions in thrombocytopenic premature infants. J Pediatr 1993;123:285-91.

40. Christensen RD. Platelet transfusion in the neonatal intensive care unit: benefits, risks, alternatives. Neonatology 2011;100:311-8.

41. Baer VL, Lambert DK, Henry E, Snow GL, Sola-Visner MC, Christensen RD. Do platelet transfusions in the NICU adversely affect survival? Analysis of 1600 thrombocytopenic neonates in a multihospital healthcare system. J Perinatol 2007;27:790-6.

42. Ferrer-Marin F, Chavda C, Lampa M, Michelson AD, Frelinger AL 3rd, Sola-Visner M. Effects of in vitro adult platelet transfusions on neonatal hemostasis. J Thromb Haemost 2011;9:1020-8. 